\title{
A Singular Values Based Newton Method for Linear Complementarity Problems*
}

\author{
Haishan Han, Yuan Li \\ College of Mathematics, Inner Mongolia University for the Nationalities, Tongliao, China \\ Email: haishanhan@sohu.com
}

Received 3 November 2015; accepted 28 December 2015; published 31 December 2015

Copyright $@ 2015$ by authors and Scientific Research Publishing Inc.

This work is licensed under the Creative Commons Attribution International License (CC BY). http://creativecommons.org/licenses/by/4.0/

c) (i) Open Access

\begin{abstract}
The existence condition of the solution of special nonlinear penalized equation of the linear complementarity problems is obtained by the relationship between penalized equations and an absolute value equation. Newton method is used to solve penalized equation, and then the solution of the linear complementarity problems is obtained. We show that the proposed method is globally and superlinearly convergent when the matrix of complementarity problems of its singular values exceeds 0; numerical results show that our proposed method is very effective and efficient.
\end{abstract}

\section{Keywords}

Linear Complementarity Problem, Nonlinear Penalized Equation, Newton Method, Singular Values

\section{Introduction}

Given a matrix $A \in R^{n \times n}$ and a vector $b \in R^{n}$, the problem of finding vectors $x \in R^{n}$ such that

$$
x \leq 0, A x-b \leq 0, x^{\mathrm{T}}(A x-b)=0
$$

is called the linear complementarity problem (LCP). We call the problem the LCP (A, b). It is well known that several problems in optimization and engineering can be expressed as LCPs. Cottle, Pang, and Stone [1] [2] provide a thorough discussion of the problem and its applications, as well as providing solution techniques.

There are a large number of general purpose methods for solving linear complementarity problems. We can divide these methods into essentially two categories: direct methods, such as pivoting techniques [1] [2], and iterative methods, such as Newton iteration [2] [3] and interior point algorithms [4].

The penalty method has been used an LCP (or, equivalently, a variational inequality) [5] [6]. The paper [7] [8] constructed a nonlinear penalized Equation (1.2) corresponding to variational inequality.

\footnotetext{
*This work supported by the Science Foundation of Inner Mongolia in China (2011MS0114).
} 
Find $x_{\lambda} \in R^{n}$ such that

$$
A x_{\lambda}+\lambda\left[x_{\lambda}\right]_{+}=b
$$

where $\lambda>1$ is the penalized parameter, $[u]_{+}=\max \{u, 0\}$.

The nonlinear penalized problems (1.2) corresponding to the linear complementarity problem (1.1), which its research has achieved good results. Wang [9] [10], Yang [11] and Li [12] [13] was extended to a general form of (1.2) to present a power penalty function

$$
A x_{\lambda}+\lambda\left[x_{\lambda}\right]_{+}^{\frac{1}{k}}=b
$$

approach to the linear complementarity problem. For the penalty Equation (1.2) Li [14] proved the solution to this equation converges to that of the linear complementarity problem when the singular values of $A$ exceed 1 and Han $[15]$ the interval matrix $\left[A, A+\lambda_{k} I\right]$ is regular. It is worth mentioning that the penalty technique has been widely used solving nonlinear programming, but it seems that there is a limited study for LCP.

Some words about our notation: $I$ refers to the identity matrix, and $y \in R^{n}$ are column vectors, $y^{T}$ refers to the transpose of the $y$, we denote by $\|y\|$ the Euclidian norm. $[y]_{+}=\max \{y, 0\}$, that generalized Jacobian $\partial[y]_{+}=D(y)$, where $D(y)$ denotes diagonal matrix, On the diagonal elements with component 1 , 0 or $\sigma \in[0,1]$ corresponding to the component of $y$ which is positive , negative or zero, respectively.

\section{Generalized Newton Method}

In this section, we will propose that a new generalized Newton method based on the nonlinear penalized Equation (1.2) for solving the linear complementarity problem.

Proposition 1 [15]. $A x_{\lambda}+\lambda\left[x_{\lambda}\right]_{+}=b$ equivalent to $0 \leq z \perp M z+q \geq 0$, where

$$
z=A x_{\lambda}-b, M=(A+\lambda I) A^{-1}, q=\lambda A^{-1} b
$$

Proposition 2. $A x_{\lambda}+\lambda\left[x_{\lambda}\right]_{+}=b$ has a unique solution if the singular values of $A$ exceed 0 .

Proof: Since the singular values of $A$ exceed 0 , then $A$ is a positive definite matrix, and $A+\lambda I$ is positive definite, then $M=(A+\lambda I) A^{-1}$ is positive definite, then $0 \leq z \perp M z+q \geq 0$ has a unique solution.

Let us note

$$
F\left(x_{\lambda}\right)=A x_{\lambda}+\lambda\left[x_{\lambda}\right]_{+}-b, \lambda>1
$$

Thus, nonlinear penalized Equation (1.2) is equivalent to the equation $F\left(x_{\lambda}\right)=0$.

A generalized Jacobian $\partial F\left(x_{\lambda}\right)$ of $F\left(x_{\lambda}\right)$ is given by

$$
\partial F\left(x_{\lambda}\right)=A+\lambda D\left(x_{\lambda}\right) .
$$

where $D\left(x_{\lambda}\right)=\partial\left[x_{\lambda}\right]_{+}$is a diagonal matrix whose diagonal entries are equal 1,0 or a real number $\sigma \in[0,1]$ depending on whether the corresponding component of $x_{\lambda}$ is positive, negative, or zero. The generalized Newton method for finding a solution of the equation $F\left(x_{\lambda}\right)=0$ consists of the following iteration:

$$
F\left(x_{\lambda}^{i}\right)+\partial F\left(x_{\lambda}^{i}\right)\left(x_{\lambda}^{i+1}-x_{\lambda}^{i}\right)=0
$$

equavelently

$$
\left[A+\lambda_{k} D\left(x_{\lambda}^{i}\right)\right] x_{\lambda}^{i+1}=q
$$

\section{Algorithm 1}

Step 1: Choose an arbitrary initial point $x_{\lambda_{0}}^{0} \in R^{n}, \varepsilon>0$ and given $\lambda_{0}>1, \mu>1, \sigma \in[0,1]$, let $k:=0$; Step 2: for the $\lambda_{k}$, computer $x_{\lambda_{k}}^{i+1}$ by solving (2.2).

Step 3: If $D\left(x_{\lambda_{k}}^{i+1}\right)=D\left(x_{\lambda_{k}}^{i}\right)$, terminate. Otherwise, $i=i+1$ go to step 2 .

Step4: If $\left\|x_{\lambda_{k}}^{i+1}\right\|<\varepsilon$, terminate, $\bar{x}=x_{\lambda_{k}}^{i+1}$ is solution of LCP. Otherwise let $\lambda_{k+1}=\mu \lambda_{k}, \quad x_{\lambda_{k+1}}^{0}=\bar{x}_{k}$ let $k:=k+1$, go to 2 . 


\section{The Convergence of the Algorithm}

We will show that the sequence $\left\{x_{\lambda_{k}}^{i}\right\}_{i=1}^{\infty}$ generated by generalized Newton iteration (2.2) converges to an accumulation point $\bar{x}_{k}$ associated with $\lambda_{k}$. First, we establish boundness of the sequence $\left\{x_{\lambda_{k}}^{i}\right\}$ for any $\lambda_{k}>0$ generated by the Newton iterates (2.2) and hence the existence of accumulation point at each generalized Newton iteration.

Theorem 1: Suppose the singular values of $M$ exceed 0 . Then, the sequence $\left\{x_{\lambda_{k}}^{i}\right\}$ generated by Algorithm 1 is bounded. Consequently, there exits an accumulation points $\bar{x}_{k}$ such that $\left[A+\lambda_{k} D\left(\bar{x}_{k}\right)\right] \bar{x}_{k}=b$.

Proof. Suppose that sequence $\left\{x_{\lambda_{k}}^{i}\right\}$ is unbounded, Thus, there exists an infinite nonzero subsequence $\left\{x_{\lambda_{k}}^{i_{j}}\right\} \subset\left\{x_{\lambda_{k}}^{i}\right\}$ such that

$$
\left\{x_{\lambda_{k}}^{i_{j}}\right\} \rightarrow \infty, D\left(x_{\lambda_{k}}^{i_{j}}\right)=\tilde{D} \text { and } \tilde{D} \in[0, I]
$$

where $\tilde{D}$ is main diagonal element of diagonal matrix which is $1,0, \sigma \in[0,1]$.

We know subsequence $\left\{\frac{x_{\lambda_{k}}^{i_{j}}}{\left\|x_{\lambda_{k}}^{i_{j}}\right\|}\right\}$ is bounded. Hence, exists convergence subsequence and assume that convergence point is $\tilde{x}$, and satisfy

$$
\left(A+\lambda_{k} \tilde{D}\right) \frac{x_{\lambda_{k}}^{i_{j}}}{\left\|x_{\lambda_{k}}^{i_{j}}\right\|}=\frac{b}{\left\|x_{\lambda_{k}}^{i_{j}}\right\|}
$$

Letting $j \rightarrow \infty$ yields

$$
\left(A+\lambda_{k} \tilde{D}\right) \tilde{x}=0,\|\tilde{x}\|=1 .
$$

Since the singular values of $A$ exceed 0 , then $A$ is regular, and $A+\lambda I$ is regular, we know that $\left(A+\lambda_{k} \tilde{D}\right)^{-1}$ is exists and hence $\tilde{x}=0$, contradicting to the fact that $\|\tilde{x}\|=1$. Consequently, the sequence $\left\{x_{\lambda_{k}}^{i}\right\}$ is bounded and there exists an accumulation point $\bar{x}_{k}$ of $\left\{x_{\lambda_{k}}^{i}\right\}$ such that

$$
\bar{x}_{k}=\left(M+\lambda_{k} D\left(\bar{x}_{k}\right)\right)^{-1} b .
$$

Under a somewhat restrictive assumption we can establish finite termination of the generalized Newton iteration at a penalized equation solution as follows.

Theorem 2: Suppose the singular values of $A$ exceed 0 and $\left\|\left(A+\lambda_{k} D\left(x_{\lambda_{k}}^{i}\right)\right)^{-1}\right\|<\frac{1}{2 \lambda_{k}}$ holds for all sufficiently large $\lambda_{k}$, then the generalized Newton iteration (2.2) linearly converges from any starting point $x_{\lambda_{k}}^{0}$ to a solution $\bar{x}_{k}$ of the nonlinear penalized Equation (1.2).

Proof. Similar to the proof of Theorem 4 in [15].

Theorem 3: Suppose the singular values of $A$ exceed 0 and $\left\|\left(A+\lambda_{k} D\left(x_{\lambda_{k}}^{i}\right)\right)^{-1}\right\|<\frac{1}{2 \lambda_{k}}$ holds, then Algorithm 1 linearly converges from any starting point $x_{\lambda_{0}}^{0}$ to a solution $x^{*}$ of the $\operatorname{LCP}(M, q)(1.1)$.

Proof. Similar to the proof of Theorem 5 in [15].

\section{Numerical Experiments}

In this section, we give some numerical results in order to show the practical performance of Algorithm 2.1 Numerical results were obtained by using Matlab R2007(b) on a 1G RAM, 1.86 Ghz Intel Core 2 processor. Throughout the computational experiments, the parameters were set as $\varepsilon=10^{-8}, \lambda_{0}=10, \mu=2$.

Example 1: The matrix $A$ of linear complementarity problem $\operatorname{LCP}(A, b)$ of as follows (This example appears in the Geiger and Kanzow [16], Jiang and Qi [17], YONG Long-quan, DENG Fang-an, CHEN Tao [18] and Han [15]): 
Table 1. Result from example 1.

\begin{tabular}{ccccc}
\hline$n$ & $x^{0}$ & $k$ & $m$ & $x^{*}=\left(\bar{x}_{1}, \bar{x}_{2}, \cdots, \bar{x}_{n}\right)^{\mathrm{T}}$ \\
\hline 6 & $(0,0,0,0,0,0)^{\mathrm{T}}$ & 3 & 2 & $(-0.3659,-0.4634,-0.4878,-0.4878,-0.4634,-0.3659)^{\mathrm{T}}$ \\
6 & $(-2,0,-2,-2,0,-2)^{\mathrm{T}}$ & 3 & 2 & $(-0.3659,-0.4634,-0.4878,-0.4878,-0.4634,-0.3659)^{\mathrm{T}}$ \\
7 & $(0,0,0,0,0,0,0)^{\mathrm{T}}$ & 3 & 2 & $(-0.3659,-0.4639,-0.4896,-0.4948,-0.4896,-0.4639,-0.3660)^{\mathrm{T}}$ \\
7 & $(1,1,1,1,1,1,1)^{\mathrm{T}}$ & 3 & 2 & $(-0.3659,-0.4639,-0.4896,-0.4948,-0.4896,-0.4639,-0.3660)^{\mathrm{T}}$ \\
\hline
\end{tabular}

Table 2. Result from example 2.

\begin{tabular}{|c|c|c|c|}
\hline$x^{0}$ & $k$ & $m$ & $\bar{x}^{*}=\left(\bar{x}_{1}, \bar{x}_{2}, \cdots, \bar{x}_{49}, \bar{x}_{50}\right)$ \\
\hline \multirow{25}{*}{$(1,-1,1,-1, \cdots, 1,-1)^{\mathrm{T}}$} & \multirow{25}{*}{1} & \multirow{25}{*}{26} & $\bar{x}_{1}=2.23517 e-9 \quad \bar{x}_{2}=-0.25$ \\
\hline & & & $\bar{x}_{3}=9.68575 e-9 \quad \bar{x}_{4}=-0.25$ \\
\hline & & & $\bar{x}_{5}=9.68575 e-9 \quad \bar{x}_{6}=-0.25$ \\
\hline & & & $\bar{x}_{7}=9.68575 e-9 \quad \bar{x}_{8}=-0.25$ \\
\hline & & & $\bar{x}_{9}=9.68575 e-9 \quad \bar{x}_{10}=-0.25$ \\
\hline & & & $\bar{x}_{11}=9.68575 e-9 \quad \bar{x}_{12}=-0.25$ \\
\hline & & & $\bar{x}_{13}=9.68575 e-9 \quad \bar{x}_{14}=-0.25$ \\
\hline & & & $\bar{x}_{15}=9.68575 e-9 \quad \bar{x}_{16}=-0.25$ \\
\hline & & & $\bar{x}_{17}=9.68575 e-9 \quad \bar{x}_{18}=-0.25$ \\
\hline & & & $\bar{X}_{19}=9.68575 e-9 \quad \bar{X}_{20}=-0.25$ \\
\hline & & & $\bar{x}_{21}=9.68575 e-9 \quad \bar{x}_{22}=-0.25$ \\
\hline & & & $\bar{x}_{23}=9.68575 e-9 \quad \bar{x}_{24}=-0.25$ \\
\hline & & & $\bar{x}_{25}=9.68575 e-9 \quad \bar{x}_{26}=-0.25$ \\
\hline & & & $\bar{x}_{27}=9.68575 e-9 \quad \bar{x}_{28}=-0.25$ \\
\hline & & & $\bar{x}_{29}=9.68575 e-9 \quad \bar{x}_{30}=-0.25$ \\
\hline & & & $\bar{x}_{31}=9.68575 e-9 \quad \bar{x}_{32}=-0.25$ \\
\hline & & & $\bar{x}_{33}=9.68575 e-9 \quad \bar{x}_{34}=-0.25$ \\
\hline & & & $\bar{x}_{35}=9.68575 e-9 \quad \bar{x}_{36}=-0.25$ \\
\hline & & & $\bar{x}_{37}=9.68575 e-9 \quad \bar{x}_{38}=-0.25$ \\
\hline & & & $\bar{x}_{39}=9.68575 e-9 \quad \bar{x}_{40}=-0.25$ \\
\hline & & & $\bar{x}_{41}=9.68575 e-9 \quad \bar{x}_{42}=-0.25$ \\
\hline & & & $\bar{x}_{43}=9.68575 e-9 \quad \bar{x}_{44}=-0.25$ \\
\hline & & & $\bar{X}_{45}=9.68575 e-9 \quad \bar{X}_{46}=-0.25$ \\
\hline & & & $\bar{X}_{47}=9.68575 e-9 \quad \bar{x}_{48}=-0.25$ \\
\hline & & & $\bar{x}_{49}=9.68575 e-9 \quad \bar{x}_{50}=-0.25$ \\
\hline$(-1,0,-1,0, \cdots,-1,0)^{\mathrm{T}}$ & 2 & 26 & Results are as above. \\
\hline$(1,1,1,1, \cdots, 1,1)^{\mathrm{T}}$ & 2 & 26 & Results are as above. \\
\hline
\end{tabular}


Table 3. Result from example 3.

\begin{tabular}{ccccc}
\hline$n$ & $k$ & $m$ & $x^{*}=\left(\bar{x}_{1}, \bar{x}_{2}, \cdots, \bar{x}_{n}\right)^{\mathrm{T}}$ \\
\hline 6 & $(1,-1,1,-1,1,-1)^{\mathrm{T}}$ & 3 & 2 & $(-6,-3,-2,-1.5,-1.2,-1)^{\mathrm{T}}$ \\
6 & $(0,0,0,0,0,0)^{\mathrm{T}}$ & 3 & 2 & $(-6,-3,-2,-1.5,-1.2,-1)^{\mathrm{T}}$ \\
8 & $(0,0,0,0,0,0,0,0)^{\mathrm{T}}$ & 3 & 2 & $(-8,-4,-2.67,-2,-1.6,-1.34,-1.14,-1)^{\mathrm{T}}$ \\
8 & $(1,-1,1,-1,1,-1,1,-1)^{\mathrm{T}}$ & 3 & 2 & $(-8,-4,-2.67,-2,-1.6,-1.34,-1.14,-1)^{\mathrm{T}}$ \\
& $(1,-1,1,-1, \cdots, 1,-1)^{\mathrm{T}}$ & 3 & 2 & $(-16,-8,-5.3,-4,-3.2,-2.67,-2.28,-2,-1.78$, \\
16 & & & $-1.6,-1.45,-1.34,-1.23,-1.14,-1.06,-1)^{\mathrm{T}}$ \\
& & & & $(-16,-8,-5.3,-4,-3.2,-2.67,-2.28,-2,-1.78$, \\
16 & $(0,0,0,0,0,0,0,0)^{\mathrm{T}}$ & 3 & 2 & $-1.6,-1.45,-1.34,-1.23,-1.14,-1.06,-1)^{\mathrm{T}}$ \\
& & & &
\end{tabular}

$$
A=\left(\begin{array}{cccccc}
4 & -1 & 0 & \cdots & 0 & 0 \\
-1 & 4 & -1 & \cdots & 0 & 0 \\
0 & -1 & 4 & \cdots & 0 & 0 \\
\vdots & \vdots & \vdots & \ddots & \vdots & \vdots \\
0 & 0 & 0 & \cdots & 4 & -1 \\
0 & 0 & 0 & \cdots & -1 & 4
\end{array}\right), \quad b=(-1,-1, \cdots,-1,-1)^{\mathrm{T}}
$$

The computational results are shown in Table 1 . This $x^{0}$ is initial point, $k$ is number of inner iterations, the outer iteration number is $m, x^{*}$ is iteration results.

Example 2: The matrix $A$ of linear complementarity problem $\operatorname{LCP}(A, b)$ of as follows:

$$
a_{i j}= \begin{cases}4, & i=j=1,2,3, \cdots, 50 \\ -1, & j=i+1, i=1,2, \cdots, 49, \quad b=(1,-1,1,-1, \cdots, 1,-1) \\ 10, & j=i-1, i=2,3, \cdots, 50\end{cases}
$$

Optimal solution of this problem is $x^{*}=(2.23517 e-9,-0.25,9.68575 e-9, \cdots,-0.25,9.68575 e-9,-0.25)^{\mathrm{T}}$. The computational results are shown in Table 2. This $x^{0}$ is initial point, $k$ is number of inner iterations, the outer iteration number is $m, x^{*}$ is iteration results.

Example 3: The matrix $A$ of linear complementarity problem $L C P(A, b)$ of as follows (This example appears in the Geiger and Kanzow [16], Jiang and Qi [17], YONG Long-quan, DENG Fang-an, CHEN Tao [18] and Han [15]):

$$
A=\operatorname{diag}\left(\frac{1}{n}, \frac{2}{n}, \cdots, 1\right), \quad b=(-1,-1,-1, \cdots, 1,-1)^{\mathrm{T}}
$$

The computational results are shown in Table 3. This $x^{0}$ is initial point, $k$ is number of inner iterations, the outer iteration number is $m, x^{*}$ is iteration results.

\section{References}

[1] Cottle, R.W., Pang, J.-S. and Stone, R.E. (1992) The Linear Complementarity Problem. Academic, Boston.

[2] Han, J.Y., Xiu, N.H. and Qi, H.D. (2006) Nonlinear Complementarity Theory and Algorithms (in Chinese). Shanghai Science and Technology Publishing House, Shanghai.

[3] Outrata, J., Kocvara, M. and Zowe, J. (1998) Nonsmooth Approach to Optimization Problems with Equilibrium Constraints: Theory, Applications and Numerical Results. Kluwer Academic Publishers, Boston. 
http://dx.doi.org/10.1007/978-1-4757-2825-5

[4] Kojima, M., Megiddo, N., Noma, T. and Yoshise, A. (1991) A Unified Approach to Interior Point Algorithms for Linear Complementarity Problems. Lecture Notes in Computer Science, Springer-Verlag, New York, Berlin. http://dx.doi.org/10.1007/3-540-54509-3

[5] Friedman, A. (1982) Variational Principles and Free-Boundary Problems. Wiley, New York.

[6] Elliott, C.M. and Ockendon, J.R. (1982) Weak and Variational Methods for Moving Boun-dary Problems. Pitman Publishing, London.

[7] Glowinski, R. (1984) Numerical Methods for Nonlinear Variational Problems. Springer-Verlag, New York, Berlin, Heideberg, Tokyo.

[8] Bensoussan, A. and Lions, J.L. (1982) Applications of Variational Inequalities in Stochastic Control. North-Holland, Amsterdam.

[9] Wang, S., Yang, X.Q. and Teo, K.L. (2006) Power Penalty Method for a Linear Complementarity Problems Arising from American Option Valuation. Journal of Optimization Theory and Applications, 129, 227-254. http://dx.doi.org/10.1007/s10957-006-9062-3

[10] Wang, S. and Huang, C.S. (2008) A Power Penalty Method for Solving a Nonlinear Parabolic Complementarity Problem. Nonlinear Analysis, 69, 1125-1137. http://dx.doi.org/10.1016/j.na.2007.06.014

[11] Wang, S. and Yang, X.Q. (2008) Power Penalty Method for Linear Complementarity Problems. Operations Research Letters, 36, 211-214. http://dx.doi.org/10.1016/j.orl.2007.06.006

[12] Li, Y., Han, H.S., Li, Y.M. and Wu, M.H. (2009) Convergence Analysis of Power Penalty Method for Three Dimensional Linear Complementarity Problem. Intelligent Information Management Systems and Technologies, 5, 191-198.

[13] Li, Y., Yang, D.D. and Han, H.S. (2012) Analysis to the Convergence of the Penalty Method for Linear Complementarity Problems. Operations Research and Management Science, 21, 129-134. (In Chinese)

[14] Li, Y., Han, H.S. and Yang, D.D. (2015) A Penalized Equation Based Generalized Newton Method for Solving Linear Complementarity Problems. Numerical Mathematics: A Journal of Chinese Universities, 37, 53-70. (In Chinese)

[15] Han, H.S. and Lan-Ying (2015) An Interval Matrix Based Generalized Newton Method for Linear Complementarity Problems. Open Journal of Applied Sciences, 5, 443-449. http://dx.doi.org/10.4236/ojapps.2015.58044

[16] Geiger, C. and Kanzow, C. (1996) On the Resolution of Monotone Complementarity Problems. Computational Optimization and Applications, 5, 155-173. http://dx.doi.org/10.1007/BF00249054

[17] Jiang, H.Y. and Qi, L.Q. (1997) A New Nonsmooth Equations Approach to Nonlinear Complementarity Problems. SIAM Journal on Control and Optimization, 35, 178-193. http://dx.doi.org/10.1137/S0363012994276494

[18] Yong, L.Q., Deng, F. and Chen, T. (2009) An Interior Point Method for Solving Monotone Linear Complementarity Problem. Journal of Mathematics, 29, 681-686. (In Chinese) 